\title{
Molecular serotyping of Haemophilus parasuis isolated from diseased pigs and the relationship between serovars and pathological patterns in Taiwan
}

\author{
Wei-Hao Lin ${ }^{1,2}$, Hsing-Chun Shih ${ }^{2}$, Chuen-Fu Lin ${ }^{3}$, Cheng-Yao Yang ${ }^{4}$, Yung-Fu Chang ${ }^{5}$, Chao-Nan Lin ${ }^{\text {Corresp., }}$ \\ 1,2, Ming-Tang Chiou ${ }^{1,2,6}$ \\ 1 Department of Veterinary Medicine, College of Veterinary Medicine, National Pingtung University of Science and Technology, Pingtung, Taiwan \\ 3 Department of Veterinary Medicine, College of Veterinary Medicine, National Chiayi University, Chiayi, Taiwan \\ 4 Graduate Institute of Veterinary Pathobiology, College of Veterinary Medicine, National Chung-Hsing University, Taichung, Taiwan \\ 5 Department of Population Medicine and Diagnostic Sciences, College of Veterinary Medicine, Cornell University, New York, United States \\ 6 Research Center for Animal Biologics, National Pingtung University of Science and Technology, Pingtung, Taiwan \\ Corresponding Author: Chao-Nan Lin \\ Email address: cnlin6@mail.npust.edu.tw
}

Background: Haemophilus parasuis (H. parasuis) is the etiological agent of Glässer's disease, and causes severe economic losses in the swine industry. Serovar classification is intended as an indicator of virulence and pathotype and is also crucial for vaccination programs and vaccine development.

According to a polysaccharide biosynthesis locus analysis, H. parasuis isolates could be classified by a molecular serotyping assay (except for serovars 5 and 12). The aim of this study was to identify $H$. parasuis isolates from diseased pigs in Taiwan by using a molecular serotyping assay and to analyze the relationship between serovars and pathological patterns.

Methods: From August 2013 to February 2017, a total of 133 isolates from 277 lesions on 155 diseased animals from 124 infected herds serotyped by multiplex PCR and analyzed with pathological data.

Results: The results showed that the dominant serovars of $H$. parasuis in Taiwan were serovars $5 / 12$ $(37.6 \%), 4(27.8 \%)$ and 13 (15\%) followed by molecular serotyping non-typable (MSNT) isolates (13.5\%), which are differentiated on a genetic basis. Nevertheless, the serovar-specific amplicons were not precisely the same sizes as previously indicated in the original publication, and MSNT isolates appeared with unexpected amplicons or lacked serovar-specific amplicons. Furthermore, most $H$. parasuis isolates were isolated from nursery pigs infected with porcine reproductive and respiratory syndrome virus. The percentage of lung lesions (30.4\%) showing $\mathrm{H}$. parasuis infection was significantly higher than that of serosal lesions.

Discussion: Collectively, the distribution of serovars in Taiwan is similar to that found in other countries, but MSNT i solates remain due to genetic variations. Furthermore, pulmonary lesions may be optimum sites for $H$. parasuis isolation, the diagnosis of Glässer's disease, and may also serve as points of origin for systemic $H$. parasuis infections in hosts. 
1

3 Wei-Hao Lin ${ }^{1,2}$, Hsing-Chun Shih ${ }^{1}$, Chuen-Fu Lin ${ }^{3}$, Cheng-Yao Yang ${ }^{4}$, Yung-Fu Chang ${ }^{5}$, Chao-

4 Nan Lin ${ }^{1,2^{*}}$, Ming-Tang Chiou ${ }^{1,2,6^{*}}$

$5{ }^{1}$ Department of Veterinary Medicine, College of Veterinary Medicine, National

6 Pingtung University of Science and Technology, Pingtung 91201, Taiwan.

$7 \quad{ }^{2}$ Animal Disease Diagnostic Center, College of Veterinary Medicine, National Pingtung

8 University of Science and Technology, Pingtung 91201, Taiwan.

$9{ }^{3}$ Department of Veterinary Medicine, College of Veterinary Medicine, National Chiayi

10 University, Chiayi 60004, Taiwan.

$11{ }^{4}$ Graduate Institute of Veterinary Pathobiology, College of Veterinary Medicine, National

12 Chung-Hsing University, Taichung, Taiwan.

13 5epartment of Population Medicine and Diagnostic Sciences, College of Veterinary Medicine,

14 Cornell University, Ithaca, NY 14850, USA.

$15{ }^{6}$ Research Center for Animal Biologics, National Pingtung University of Science and

16 Technology, Pingtung, Taiwan.

18 *Corresponding Author:

19 Chao-Nan Lin ${ }^{1,2}$

20 VM208, 1, Shuefu Road, Neipu, Pingtung 91201, Taiwan

21 Email address: cnlin6@,mail.npust.edu.tw

22 Ming-Tang Chiou ${ }^{1,2,6}$

23 VM212, 1, Shuefu Road, Neipu, Pingtung 91201, Taiwan

24 Email address: mtchiou@,mail.npust.edu.tw 


\section{Abstract}

27 Background: Haemophilus parasuis (H. parasuis) is the etiological agent of Glässer's disease,

28 and causes severe economic losses in the swine industry. Serovar classification is an indicator of

29 virulence and pathotype and is also crucial for vaccination programs and vaccine development.

H. parasuis isolates can be classified by a molecular serotyping assay except for serovars 5 and

12. The aim of this study was to serotype $H$. parasuis isolates from diseased pigs in Taiwan by using a molecular serotyping assay and to analyze the relationship between serovars and pathological patterns.

Methods: From August 2013 to February 2017, a total of 155 diseased animals from 124 infected herds were examined for infection with $H$. parasuis. One hundred thirty three isolates of H. parasuis were recovered and serotyped by multiplex PCR and correlated with pathological

37 lesions.

Results: The dominant $H$. parasuis were serovars 5/12 (37.6\%), $4(27.8 \%)$ and $13(15 \%)$ followed by molecular serotyping non-typable (MSNT) isolates (13.5\%). The serovar-specific amplicons were not precisely the same sizes as previously indicated in the original publication, and MSNT isolates appeared with unexpected amplicons or lacked serovar-specific amplicons. Most $H$. parasuis isolates were isolated from nursery pigs infected with porcine reproductive and respiratory syndrome virus. The percentage of lung lesions (30.4\%) showing $H$. parasuis infection was significantly higher than that of each serosal lesions.

Discussion: Collectively, the distribution of $H$. parasuis serovars in Taiwan is similar other countries, but MSNT isolates remain due to genetic variations. Our data suggests those prevalent serovar isolates prefer to cause both serosal and pulmonary lesions rather and only pulmonary lesions. Pulmonary lesions are optimum sites for $H$. parasuis isolation and may also serve as 
49 points of origin for systemic $H$. parasuis infections in hosts.

50 Keywords: Haemophilus parasuis, Glässer's disease, polyserositis, serotyping

51

\section{Introduction}

53

54

55

56

57

Haemophilus parasuis (H. parasuis), a part of normal upper respiratory microbiota, is the etiological agent of Glässer's disease which induces sudden death, polyserositis, polyarthritis, meningitis and poor production performance, resulting severe economic losses in the swine industry (Amano et al. 1994; Moller \& Kilian 1990; Vahle et al. 1997; Zhang et al. 2014). Vaccination is an effective strategy for preventing increased mortality and economic losses caused by virulent $H$. parasuis (Miniats et al. 1991a; Smart \& Miniats 1989). However, only partial protection is observed with heterologous $H$. parasuis strain challenges due to poor crossprotection (Miniats et al. 1991b; Nielsen 1993; Takahashi et al. 2001). Thus, serotyping of $H$. parasuis is very important, not only for epidemiological research but also for choosing efficacious inactivated whole-cell bacterial vaccines.

Fifteen serovars, conventional serotyping cross-reactive (CSCR) and non-typable (CSNT) isolates of $H$. parasuis have been described and demonstrated by gel immunodiffusion assay (GID) (Kielstein \& Rapp-Gabrielson 1992). Due to the persistence of cross-reactivity or nonreaction to antisera, there are still approximately $15 \%$ to $40 \%$ CSCR and CSNT isolates reported in a variety of countries by GID (Table S1) (Blackall et al. 1996; Cai et al. 2005; Castilla et al. 2012; Del Rio et al. 2003; Kielstein \& Rapp-Gabrielson 1992; Luppi et al. 2013; Ma et al. 2016; Oliveira et al. 2003; Rapp-Gabrielson \& Gabrielson 1992; Rubies et al. 1999; Tadjine et al. 2004). Despite using an indirect hemagglutination assay (IHA) designed to reduce the proportion of CSCR isolates, $7.5 \%$ to $18 \%$ of isolates are still untypable (Table S1) (Angen et al. 2004; Cai et al. 2005; Del Rio et al. 2003; Dijkman et al. 2012; Howell et al. 2015). This phenomenon 
73 makes it more difficult to conduct an effective vaccination program against $H$. parasuis.

74 Conventional serotyping is used extensively (Kielstein et al. 1991; Morozumi \& Nicolet

75 1986; Rapp-Gabrielson \& Gabrielson 1992). The Kielstein-Rapp-Gabrielson (KRG) scheme

76 recognizes 15 serovars of $H$. parasuis on the basis of a GID test with specific rabbit antisera and

77 the authors noted a correlation between serovar and virulence (Kielstein \& Rapp-Gabrielson

78 1992). According to serotyping results, serovar 4 tends to be found in pulmonary infections;

79 CSNT and CSCR isolates are mainly found in systemic infections (Angen et al. 2004).

80 Unfortunately, others report little correlation between serovar and virulence as isolates in the

81 same serovar often exhibit different virulence levels (Aragon et al. 2010; Olvera et al. 2007).

82 Previous studies established the serovar and pathotype of $H$. parasuis are based on

83 differences at the genome level (Brockmeier et al. 2014; Howell et al. 2013; Howell et al. 2017).

84 A multiplex PCR (mPCR) based on a polysaccharide biosynthesis locus analysis was employed

85 to molecularly serotype $H$. parasuis serovars (Howell et al. 2015). As a result, 14 of 15 serovars

86 of $H$. parasuis (serovars 5 and 12 could not be differentiated) were identified using this assay

87 (Howell et al. 2015) Using the molecular typing assay many of the CSNT and CSCR isolates

88 were successfully typed in a recognized serovar.

89 Although Glässer's disease is common in Taiwan, serotyping of pathogenic H. parasuis

90 isolates from Taiwanese pigs is not clear. The principal aim of this study was to molecularly

91 serotype H. parasuis isolated from Taiwanese diseased pigs, and correlate serovars with

92 pathological patterns.

94 Materials \& Methods

95 Bacterial isolate collection and identification

96 H. parasuis field isolates were collected from diseased pig herds between August 2013 and 
97 February 2017 in Taiwan (Table S2). Lesions suspected of being caused by H. parasuis in

98 diseased pigs were located in the meninges, pleura, pericardia, peritonea, synovial cavities of

99 joints and lungs. Lesions were swabbed and plated on chocolate agar (at $37^{\circ} \mathrm{C}, 5 \% \mathrm{CO}_{2}, 18$ to 72

100 hours for growth rate variation for various isolates), blood agar (at $37^{\circ} \mathrm{C}, 16$ to 24 hours) and

101 MacConkey agar (at $37^{\circ} \mathrm{C}, 16$ to 24 hours). The bacterial isolates were identified by colony

102 morphology, Gram stain (Gram negative bacillus), nicotinamide adenine dinucleotide (NAD)

103 dependence (only growing on chocolate agar) and virulence-associated trimeric autotransporter

104 group 3 colony PCR (Pina et al. 2009).

105 Molecular serotyping mPCR

106 The molecular serotyping assay for H. parasuis isolates was modified from a previously

107 published method (Howell et al. 2015). The sp-sp amplicon was used as an internal control. A

108 loopful of bacteria from a passaged plate of pure culture was resuspended in $30 \mu \mathrm{L}$ ultrapure

$109 \mathrm{H}_{2} \mathrm{O}$, which was heated to $100^{\circ} \mathrm{C}$ for $30 \mathrm{~min}$ then centrifuged at $4,000 \mathrm{x}$ g for $1 \mathrm{~min}$. The

110 supernatant was used in the $\mathrm{mPCR}$ reaction. Isolates from various lesions or pigs from the same

111 herd were serotyped. If they belonged to same serovar, they were considered one isolate.

112 Each PCR reaction was performed in a total volume of $30 \mu \mathrm{L}$ containing ultrapure $\mathrm{H}_{2} \mathrm{O}, 1 \mathrm{x}$

113 DreamTaq buffer, $250 \mu \mathrm{M}$ dNTP, $0.2 \mu \mathrm{M}$ concentrations of forward and reverse serovar-specific

114 primers, $0.04 \mu \mathrm{M}$ concentrations of forward and reverse species-specific primers, $1.25 \mathrm{U}$ of

115 DreamTaq DNA polymerase (Thermo Fisher Scientific, Waltham, MA, USA) and $1 \mu \mathrm{L}$ of

116 supernatant. The thermocycling conditions consisted of 5 min at $94^{\circ} \mathrm{C}, 30$ cycles of $30 \mathrm{sec}$ at

$11794^{\circ} \mathrm{C}, 30 \mathrm{sec}$ at $58^{\circ} \mathrm{C}$ and $1 \mathrm{~min}$ at $72^{\circ} \mathrm{C}$, and then a final extension at $72^{\circ} \mathrm{C}$ for $5 \mathrm{~min}$. The

118 molecular serotyping mPCR amplicons were stained with ethidium bromide and analyzed using

119 a 20-cm-long 2\% agarose gel. A 50-bp DNA ladder RTU (GeneDireX, Las Vegas, NV, USA)

120 and Bio-1D software (Vilber Lourmat, Collégien, France) were used to estimate molecular size. 
121 The electrophoresis conditions were an electric field $6 \mathrm{~V} / \mathrm{cm}(300 \mathrm{~V}, 50$-cm full-length electric

122 field) and $3 \mathrm{hr}$. The results were confirmed by twice repeating tests.

123 Sequencing and analysis of unexpected PCR-amplified products

124 Unexpected amplicons of the molecular serotyping mPCR products were cloned using a TA

125 cloning kit (Yeastern Biotech Co., Ltd. Taipei, Taiwan) and sequenced using an automated DNA

126 sequencer (ABI 3730XL, USA). Sequence data were analyzed using MEGA7 (Molecular

127 Evolutionary Genetics Analysis Version 7.0) software and BLAST (Basic Local Alignment

128 Search Tool) database.

129 Pathological examination

130 Cases of sick animals or fresh, complete carcasses were subjected to necropsy for gross

131 morphological examinations and H\&E staining. Histopathological examination focused

132 primarily on meningeal, pleural, pericardial, peritoneal, and synovial cavities of joints, and lungs.

133 Typical meningeal gross lesions were characterized by yellow to white exudate accumulation in

134 the subarachnoid space, on pia mater and in the sulci (Fig. S1). Meningeal histopathological

135 lesions were principal neutrophils and few mononuclear inflammatory cells infiltration on pia

136 mater with fibrin and cellular debris deposit (Fig. S2). Serosal lesions were characterized by

137 yellow to white exudate accumulation in pleural, pericardial, abdominal and joint synovial

138 cavities and yellow to white fibrin covering the visceral and parietal serosa (Fig. S3; Fig. S4; Fig.

139 S5). The histopathological lesions of serositis were principal neutrophils and few mononuclear

140 inflammatory cells infiltration with fibrin deposit (Fig. S6). In typical cases, H. parasuis resulted

141 in bronchopneumonia with numerous neutrophils, mononuclear inflammatory cells, erythrocytes,

142 cellular debris and fibrinous exudate accumulation in alveoli (Fig. S7; Fig. S8). Due to disease

143 duration, lesions varied in field. Other lesions infected $H$. parasuis were also involved to

144 determine pathological patterns, including chronic fibrous serositis with angiogenesis and 
145 mononuclear inflammatory cells infiltration (Fig. S9), and meningitis with principal

146 macrophages infiltration (Fig. S10).

147 Detection of porcine reproductive and respiratory syndrome virus

148 Nucleic acid extraction of pulmonary tissue was performed on a MagNA Pure LC 2.0 by

149 using the MagNA Pure LC total nucleic acid isolation kit (Roche Applied Science, Indianapolis,

150 IN, USA). Following cDNA synthesis was using PrimeScript ${ }^{\mathrm{TM}}$ RT reagent kits (Takara, Kyoto,

151 Japan). Porcine reproductive and respiratory syndrome virus (PRRSV) reverse transcription real-

152 time PCR was performed as previously described (Lin et al. 2013).

153 Statistical analysis

154 Fisher's exact test was used to compare the frequency of $H$. parasuis infected lesions and

155 the percentage of various lesion patterns using GraphPad Prism software (GraphPad Software,

156 La Jolla, CA, USA). Variables were considered significant at a 0.05 level (two-sided).

\section{Results}

\section{H. parasuis isolates, origins and pathological lesion patterns}

160 One hundred thirty three isolates of H. parasuis were isolated from August 2013 to

161 February 2017. The isolates were taken from 277 lesions on 155 diseased animals from 124

162 infected herds. Isolates from a herd serotyped as a single serovar were calculated as one isolate.

163 Of $155 H$. parasuis cases, 12 cases $(7.7 \%)$ belonged to suckling pigs ( $\leq 3$-week-old), 133 cases

$164(85.2 \%)$ belonged to nursery pigs (4- to 12 -week-old), 7 cases (4.5\%) belonged to growing pigs

165 (13- to 26-week-old) and one case belonged to a breeding boar. Age information for two cases

166 was unknown. Eighty-six cases (55.5\%) had H. parasuis isolated from lung lesions with or

167 without serosal lesions.

168 One hundred eight animals were necropsied with complete pathological examination and 
169 further correlated to pathological pattern and isolation proportion (Table S3). Of the H. parasuis

170 infected animals, 54.6\% had serositis and pulmonary tissue lesions, $41.7 \%$ had serosal lesions

171 only, and $3.7 \%$ displayed only pulmonary lesions (Fig. 1).

172 One hundred six cases (98.1\%) had bronchopneumonia, 64 cases $(59.3 \%)$ displayed $H$.

173 parasuis positive lung lesions. Seventy-eight cases (72.2\%) registered as positive for PRRSV via

174 reverse transcription real-time PCR screening. The proportion of $204 \mathrm{H}$. parasuis infected

175 lesions from 108 animals with complete pathological examination were meninges (10.3\%),

176 pleura (20.1\%), pericardium (16.2\%), peritoneum (13.7\%), joint synovial cavity (9.3\%) and lung

177 (30.4\%) (Fig. 2). The percentage of lung lesions showing H. parasuis infection was significantly

178 higher than the percentage of serosal lesions $(P<0.05)$.

\section{Serovar distribution by molecular serotyping assay}

180 Of the 133 isolates, 91 (68.94\%) isolates were typed using molecular serotyping mPCR.

181 The most common serovars were serovar 5/12 (38.2\%) and serovar $4(27.5 \%)$ followed by

182 serovar $14(2.3 \%)$, serovar $1(0.8 \%)$, serovar $2(0.8 \%)$ and serovar $9(0.8 \%)$ (Fig. 3). However,

183 the product sizes of the serovar-specific primers analyzed by Bio-1D software were varied from

184 the original publication (Howell et al. 2015). Furthermore, there were still 41 isolates (29.8\%)

185 classified as MSNT; these were divided into four groups based on the appearance of unexpected

186 amplicons or the lack of serovar-specific amplicons. Eighteen isolates (13\%) positive for a

187 species-specific (sp-sp) marker were categorized as MSNT group 1. Nineteen isolates (14.5\%)

188 were placed in MSNT group 2; these displayed amplicons of 300, 830, and $1000 \mathrm{bp}$. Two

189 isolates (1.5\%) which showed unexpected amplicons at 500 and $660 \mathrm{bp}$ were categorized as

190 MSNT group 3. One isolate (0.8\%), showing an amplicon of $300 \mathrm{bp}$ was categorized as an

191 MSNT group 4 isolate (Fig. 3; Fig. S11; Fig. S12; Fig. S13; Fig. S14; Fig. S15).

\section{Identification of serovar-specific amplicons}


193 The amplicons generated from molecular serotyping mPCR analyzed using Bio-1D

194 software were not precisely the same sizes as previously indicated in the original description of

195 this assay (Howell et al. 2015). The product size of a specific amplicon found in serovar 4 was

196 mentioned at $320 \mathrm{bp}$ in the original publication but the PCR run generated an amplicon of nearly

$197350 \mathrm{bp}$ which might be confused with serovar 6 . In serovar 5, the PCR results generated an

198 amplicon larger than $450 \mathrm{bp}$ mentioned in the original publication which might be confused with

199 serovar 7. The product size of serovar 9 serovar-specific primers, mentioned at $710 \mathrm{bp}$ in the

200 original publication, was smaller than the 700 bp ladder marker and might be confused with

201 serovar 8. In light of these conflicting results, the isolates were serotyped again to confirm the

202 sizes, and the amplicons were subsequently sequenced. Comparisons of the molecular serotyping

203 original publication described, Bio-1D software analyzed, and BLAST product sizes are shown

204 in Table 1 (Howell et al. 2015). The product sizes analyzed by Bio-1D software, BLAST and

205 sequence are more consistent.

206 The unexpected PCR products of the MSNT isolates were cloned for sequencing (Table 2).

207 The MSNT group 2 amplicon was $297 \mathrm{bp}$; this product was generated with a serovar 13 specific

208 forward and a serovar 14 specific reverse primer pair targeting gltP gene as a marker of serovar

20913 in a polysaccharide biosynthesis locus. These primers were paired because the target

210 sequences in the respective serovars shared homologous segments. The other PCR generated an

211 amplicon product of the MSNT group 2 isolate determined to be $836 \mathrm{bp}$, and was identified as a

212 serovar 13 specific product. The Bio-1D software measured a $500 \mathrm{bp}$ product of the MSNT

213 group 3 isolate as $499 \mathrm{bp}$; this amplicon was identified as a serovar 7 specific product. The 300

214 bp product found in the MSNT group 4 isolate, (sequencing results indicated it was $297 \mathrm{bp}$ ) was

215 generated by pairing a serovar 13 specific forward primer with a serovar 14 specific reverse

216 primer. This result was the same as that generated using the same primer pair of DNA isolated 
217 from MSNT group 2.

\section{Serovar distribution based on molecular serotyping assay and sequencing}

219 The molecular serotyping assay combined with sequencing results reduced the percentage

220 of isolates classified as MSNT from 30.1\% to $13.5 \%$ (Fig. 3). The dominant serovars were

221 serovar 5/12 (37.6\%), serovar $4(27.8 \%)$ and serovar $13(15 \%)$ followed by serovar $14(2.3 \%)$,

222 serovar $7(1.5 \%)$, serovar $1(0.8 \%)$, serovar $2(0.8 \%)$ and serovar $9(0.8 \%)$. Combining the

223 sequencing results showed that serovar 13 is a common serovar.

\section{Relationship between pathological lesion patterns and serovars}

The distribution of $H$. parasuis serovars in lesions from necropsied animals were serovar 5 (42.6\%), serovar 4 (21.3\%), serovar $13(20.4 \%)$, and MSNT group $1(13 \%)$. These categories were further subdivided into animals displaying both serosal and pulmonary lesions, those with of lesions vs. serovars, and the pattern of lesions in infected animals were showed in Fig. 1 and Table S4. Necropsied animals with both serosal and pulmonary lesions were the most frequent; animals with pulmonary lesions alone were the least frequent $(p<0.0001)$. Serovars 4 and 5/12 showed similar results, the MSNT group 1 both serosal and pulmonary lesions were more frequent than serosal lesions alone. Serovar 13 had more serosal lesions than the combination of serosal and pulmonary lesions.

Nine herds $(7.3 \%)$ had populations infected with two $H$. parasuis serovars. One herd contained a population with lesions infected with serovars 1 and 4 . Three herds were infected with serovars 4 and 5. Serovar 5, 13 and 5, 14 co-infections were seen in single herds. The infected lesions were located in animals displaying a variety of tissue lesion patterns. Other 4

239 herds contained individuals co-infected with two $H$. parasuis serovars. One clinical case showed pleural and pulmonary lesions coinfected with $H$. parasuis serovars 4 and 7 , respectively. A 
241 separate herd contained one case of pulmonary lesions with serovar 5, as well as pleural,

242 pericardial, and peritoneal lesions infected with $H$. parasuis serovar 13. One case was co-

243 infected with serovars 5 (pulmonary) and 13 (pleura and pericardium). A fourth case showed

244 coinfection with serovar 4 and an MSNT group 1 isolate taken from separate pulmonary lesions.

246 Discussion

247 This is the first study describing serovars of $H$. parasuis defined by molecular serotyping in

248 Taiwan. The most common serovars are serovar 5/12, 4 and 13, followed by MSNT isolates.

249 Even though serotyping assays vary, the serovar population profile of $H$. parasuis in Taiwan is

250 similar to profiles described in several other studies (Table S1) (Angen et al. 2004; Blackall et al.

251 1996; Cai et al. 2005; Castilla et al. 2012; Del Rio et al. 2003; Dijkman et al. 2012; Howell et al.

252 2015; Kielstein \& Rapp-Gabrielson 1992; Luppi et al. 2013; Ma et al. 2016; Oliveira et al. 2003;

253 Rapp-Gabrielson \& Gabrielson 1992; Rubies et al. 1999; Tadjine et al. 2004). Most commercial

254 H. parasuis vaccines are inactivated vaccines, which provide protection against the same serovar

255 but are unable to provide protection from challenge using different serovars (Miniats et al.

256 1991b; Nielsen 1993; Smart \& Miniats 1989; Takahashi et al. 2001). Candidate serovar

257 composition in $H$. parasuis vaccine determines the success of a vaccine strategy against $H$.

258 parasuis (Takahashi et al. 2001). Therefore, the distribution of serovars in herds is an important

259 factor in outlining vaccination strategies and vaccine developments aimed at the prevention and

260 control of Glässer's disease.

261 IHA was applied to $H$. parasuis serovar differentiation to decrease the proportion of $H$.

262 parasuis isolates classified as CSCR (Cai et al. 2005; Del Rio et al. 2003). De-encapsulation due

263 to multiple passages results in non-reaction with antisera and cross reactivity of isolate antigens

264 to diagnostic (immune-based) test reagents are the primary factors behind CSNT and CSCR $H$. 
265 parasuis isolates, respectively (Cai et al. 2005; Kielstein \& Rapp-Gabrielson 1992; Oliveira et al. 266 2003; Rapp-Gabrielson \& Gabrielson 1992; Turni \& Blackall 2005). The presence of CSNT and

267 CSCR isolates confounds epidemiological surveys used to assess $H$. parasuis isolate population 268 profiles, and impairs efforts to generate effective vaccines against this pathogen. The correlation

269 between the capsule and serovar of $H$. parasuis is well established; a multiplex serotyping PCR 270 was developed with this in mind (Howell et al. 2015; Howell et al. 2013). This protocol can be

271 employed to type isolates previously classified as CSNT and CSCR via traditional

272 (immunological) methods. The mPCR serotyping reduced the incidence (percentage) of CSNT

273 and CSCR H. parasuis. Molecular serotyping has not completely eliminated the issue of CSNT

274 and CSCR isolates. One reason may be the sequence similarity of different serovar-specific

275 primers and serovar-specific products. Another factor may be deletions and/or unknown

276 sequences within certain antigenic markers (Ma et al. 2016). This underscores the importance of

277 MSNT isolate whole-genome sequencing for in silico serotyping and improving the molecular

278 serotyping assay. Emergence of MSNT isolates by the molecular serotyping assay may be due to

279 insufficient or incomplete sequence data for $H$. parasuis from Asia. When this assay was

280 developed, there were only nine Asian H. parasuis isolates in a 212-isolate database (7 from

281 Japan, 2 from China). Investigating the sequences and gene composition of Asian H. parasuis

282 isolate capsule loci may be key for assaying and serotyping MSNT isolates. Besides, absence of

283 serovar-specific markers in polysaccharide biosynthesis loci in MSNT isolates may create

284 antigenic variation impairing vaccine strategies. Therefore, it is also important to study the

285 antigenic variation due to gene mutation and/or absence in polysaccharide biosynthesis loci in 286 the future.

287 Thus far, molecular serotyping has been challenging as there are 15 serovars, making it 288 difficult to design serovar-specific primers yielding differential results. Some primer pairs 
289 produce amplicons from different $H$. parasuis serovars that vary by less than 20 bp-a difference

290 that is hardly detected especially when the amplicon size is larger than $600 \mathrm{bp}$. In our study,

291 electrophoresis using longer agarose gels was performed to enhance the ability of the procedure

292 to discriminate DNA fragment sizes. Bio-1D software was applied to more accurately measure

293 product size based on the intensity of the bands and decrease human operation error. In the case

294 of molecular serotyping tests resulting in ambiguities, serovar-specific primer pairs may be used

295 (in simplex PCR format) to confirm or classify hard-to-identify serovars. According to sequence

296 analysis, the product sizes described in the original publication were not accurate (Howell et al.

297 2015). The corrected product sizes are important to avoid mis-serotyping.

298 According to a previous study, pigs were infected with $H$. parasuis serovars 1, 5, 10, 12, 13,

299 and 14 showed high mortality. Pigs challenged with serovars $2,4,8$, and 15 showed

300 polyserositis. Pigs inoculated with serovars 3, 6, 7, 9, and 11 resulted in no clinical symptoms or

301 lesions (Kielstein \& Rapp-Gabrielson 1992). Serovars 5/12, 4, 13 and 7 are the most common

302 serovars in most countries worldwide (Table S1). Previous study has showed serovar 4 and 13

303 have a higher prevalence in systemic infection than in respiratory disease only (Luppi et al.

304 2013). Our data also showed similar results of serovar 4, 5/12, 13 and MSNT isolates. There may

305 be some correlation between serovar and virulence because serovars are defined by capsule

306 which can directly interact with host cells and has been proven to be a key virulence factor

307 relating to phagocytosis resistance (Olvera et al. 2009). Besides, it should be considered if the

308 impact of some isolates resulting in only pulmonary lesions are underestimate in field due to

309 absence of serositis and typical pulmonary lesions. The role and economic impact of $H$. parasuis

310 in pulmonary infection animals related to porcine respiratory disease complex in field is also

311 worthy of investigation in the future. In our study, serovars 7 and 9 caused serositis with or

312 without respiratory lesions. Serovar 5 isolate was isolated from an animal with only 
313 bronchopneumonia lesions and another with lesions in both the serosa and lung tissues in the

314 same herd. Therefore, the results show clinical manifestations of Glässer's disease are influenced

315 by multiple factors, including host, stress, environment, co-infection with different serovars or

316 other pathogens, and gene differences between infecting $H$. parasuis isolates (Boerlin et al. 2013;

317 Howell et al. 2014; Li et al. 2009). In general, most Glässer's disease cases in nursery pigs were

318 co-infected with PRRSV in our data. This may be because PRRSV can cause

319 immunosuppression by reducing non-specific bactericidal activity of pig alveolar macrophages

320 and stimulating interleukin-10 production, which down-regulates inflammatory cytokines (Drew

321 2000; Flores-Mendoza et al. 2008; Suradhat \& Thanawongnuwech 2003). The previous studies

322 have showed PRRSV does not result in an increased Glässer's disease by experimental challenge

323 (Segales et al. 1999; Solano et al. 1997). However, significant association between H. parasuis

324 and PRRSV in field was reported (Palzer et al. 2015). Recently studies also showed PRRSV can

325 induce bronchopneumonia with Bordetella bronchiseptica which is a part of normal upper

326 respiratory microbiota and predispose to colonization with $H$. parasuis (Brockmeier 2004;

327 Brockmeier et al. 2001). Co-infection of pig alveolar macrophages with PRRSV and H. parasuis

328 leads to pro-inflammatory mediated immunopathology by synergistic effect (Kavanova et al.

329 2015; Li et al. 2017). In the future, the synergistic effect between PRRSV and H. parasuis

330 resulting in economic losses in field is worthy of further investigation. Other factors also interact

331 with $H$. parasuis including the stress of weaning and maternal antibody reduction. However,

332 highly virulent $H$. parasuis isolates might be considered primary pathogens (Aragon et al. 2012).

333 In our study, some $H$. parasuis isolates caused serositis and sudden death without co-infection,

334 even in growing pigs and breeding boars.

335 Previous studies showed $H$. parasuis can access the blood stream through invasion of the 336 mucosal surface in the nasal cavity (Vahle et al. 1997). In our study, pulmonary lesions showed 
337 higher pathogenic $H$. parasuis infection rates than serosal lesions. These results are in

338 accordance with a previous study from the Netherlands (Dijkman et al. 2012). H. parasuis

339 invasion and survival in lung tissue is likely a key feature for the onset of disease (Olvera et al.

340 2009; Vahle et al. 1995). Our results show H. parasuis infected animals with lesions found in

341 dual anatomical locations (pulmonary and serosal) occur at a higher rate than infected animals

342 with lesions located in only one tissue type. Previous studies also mentioned lung is one of the

343 most successful sites for acute (serovar 12) and subacute (serovar 4) isolate recovery (Turni \&

344 Blackall 2007). Therefore, lung is an important origin for $H$. parasuis isolation and a target organ

345 for Glässer's disease diagnosis. Pulmonary infections may be an important step for H. parasuis

346 systemic infections.

347 Others have reported isolation of multiple isolates from single pig farms (Cerda-Cuellar et

348 al. 2010; Oliveira et al. 2003; Olvera et al. 2006a; Olvera et al. 2006b). Our results also show

349 different serovars cause disease in a single herd, or even in a single animal, although the latter

350 scenario is fairly uncommon. In most situations, Glässer's disease is caused by one isolate

351 (Rafiee et al. 2000), but several isolates may be present at a given farm (Turni \& Blackall 2010).

352 Therefore, it would be useful to develop a universal vaccine against multiple serovars. The

353 possibility of cross talk between different pathogenic $H$. parasuis isolates at a given site may be

354 worthy of investigation.

355

356 Conclusions

357 Our study shows the dominant serovars of $H$. parasuis in Taiwan are serovars 5/12, 4 and

358 13, followed by MSNT isolates. Proportions of isolates in those serovars resulting in both serosal

359 and pulmonary lesions are significantly higher than pulmonary lesion. Pulmonary lesions may be 
360 most important for $H$. parasuis isolation, and may serve as points of origin for systemic $H$.

361 parasuis infections in hosts.

362

363

\section{References}

364

Amano H, Shibata M, Kajio N, and Morozumi T. 1994. Pathologic observations of pigs

365

366

367

368

369

370

371

372

373

374

375

376

377

378

379

380

381

382

383

384

385

386

387

388

389

390

391

392

393 intranasally inoculated with serovar 1, 4 and 5 of Haemophilus parasuis using immunoperoxidase method. Journal of Veterinary Medical Science 56:639-644. 10.1292/jvms.56.639

Angen O, Svensmark B, and Mittal KR. 2004. Serological characterization of Danish Haemophilus parasuis isolates. Veterinary Microbiology 103:255-258. 10.1016/j.vetmic.2004.07.013

Aragon V, Cerda-Cuellar M, Fraile L, Mombarg M, Nofrarias M, Olvera A, Sibila M, Solanes D, and Segales J. 2010. Correlation between clinico-pathological outcome and typing of Haemophilus parasuis field strains. Veterinary Microbiology 142:387-393. 10.1016/j.vetmic.2009.10.025

Aragon V, Segalés J, and Oliveira S. 2012. Glässer's Disease. In: Zimmerman JJ, Karriker LA, Ramirez A, Schwartz KJ, and Stevenson GW, eds. Diseases of Swine. 10 ed. Chichester: Wiley-Blackwell, 760-769.

Blackall PJ, Rapp-Gabrielson VJ, and Hampson DJ. 1996. Serological characterisation of Haemophilus parasuis isolates from Australian pigs. Australian Veterinary Journal 73:93-95.

Boerlin P, Poljak Z, Gallant J, Chalmers G, Nicholson V, Soltes GA, and MacInnes JI. 2013. Genetic diversity of Haemophilus parasuis from sick and healthy pigs. Veterinary Microbiology 167:459-467. 10.1016/j.vetmic.2013.07.028

Brockmeier SL. 2004. Prior infection with Bordetella bronchiseptica increases nasal colonization by Haemophilus parasuis in swine. Veterinary Microbiology 99:75-78. 10.1016/j.vetmic.2003.08.013

Brockmeier SL, Palmer MV, Bolin SR, and Rimler RB. 2001. Effects of intranasal inoculation with Bordetella bronchiseptica, porcine reproductive and respiratory syndrome virus, or a combination of both organisms on subsequent infection with Pasteurella multocida in pigs. American Journal of Veterinary Research 62:521-525.

Brockmeier SL, Register KB, Kuehn JS, Nicholson TL, Loving CL, Bayles DO, Shore SM, and Phillips GJ. 2014. Virulence and draft genome sequence overview of multiple strains of the swine pathogen Haemophilus parasuis. PloS One 9:e103787. 
394

395

396

397

398

399

400

401

402

403

404

405

406

407

408

409

410

411

412

413

414

415

416

417

418

419

420

421

422

423

424

425

426

427

428

429

10.1371/journal.pone.0103787

Cai X, Chen H, Blackall PJ, Yin Z, Wang L, Liu Z, and Jin M. 2005. Serological characterization of Haemophilus parasuis isolates from China. Veterinary Microbiology 111:231-236. 10.1016/j.vetmic.2005.07.007

Castilla KS, de Gobbi DD, Moreno LZ, Paixao R, Coutinho TA, dos Santos JL, and Moreno AM. 2012. Characterization of Haemophilus parasuis isolated from Brazilian swine through serotyping, AFLP and PFGE. Research in Veterinary Science 92:366-371. 10.1016/j.rvsc.2011.04.006

Cerda-Cuellar M, Naranjo JF, Verge A, Nofrarias M, Cortey M, Olvera A, Segales J, and Aragon V. 2010. Sow vaccination modulates the colonization of piglets by Haemophilus parasuis. Veterinary Microbiology 145:315-320. 10.1016/j.vetmic.2010.04.002

Del Rio ML, Gutierrez CB, and Rodriguez Ferri EF. 2003. Value of indirect hemagglutination and coagglutination tests for serotyping Haemophilus parasuis. Journal of Clinical Microbiology 41:880-882. 10.1128/JCM.41.2.880-882.2003

Dijkman R, Wellenberg GJ, van der Heijden HM, Peerboom R, Olvera A, Rothkamp A, Peperkamp K, and van Esch EJ. 2012. Analyses of Dutch Haemophilus parasuis isolates by serotyping, genotyping by ERIC-PCR and Hsp60 sequences and the presence of the virulence associated trimeric autotransporters marker. Research in Veterinary Science 93:589-595. 10.1016/j.rvsc.2011.10.013

Drew TW. 2000. A review of evidence for immunosuppression due to porcine reproductive and respiratory syndrome virus. Veterinary Research 31:27-39. 10.1051/vetres:2000106

Flores-Mendoza L, Silva-Campa E, Resendiz M, Osorio FA, and Hernandez J. 2008. Porcine reproductive and respiratory syndrome virus infects mature porcine dendritic cells and up-regulates interleukin-10 production. Clinical and Vaccine Immunology 15:720-725. 10.1128/CVI.00224-07

Howell KJ, Peters SE, Wang J, Hernandez-Garcia J, Weinert LA, Luan SL, Chaudhuri RR, Angen O, Aragon V, Williamson SM, Parkhill J, Langford PR, Rycroft AN, Wren BW, Maskell DJ, Tucker AW, and Consortium BRT. 2015. Development of a multiplex PCR assay for rapid molecular serotyping of Haemophilus parasuis. Journal of Clinical Microbiology 53:3812-3821. 10.1128/JCM.01991-15

Howell KJ, Weinert LA, Chaudhuri RR, Luan SL, Peters SE, Corander J, Harris D, Angen O, Aragon V, Bensaid A, Williamson SM, Parkhill J, Langford PR, Rycroft AN, Wren BW, Holden MT, Tucker AW, Maskell DJ, and Consortium BT. 2014. The use of genome wide association methods to investigate pathogenicity, population structure and serovar in Haemophilus parasuis. BMC Genomics 15:1179. 10.1186/1471-2164-15-1179

Howell KJ, Weinert LA, Luan SL, Peters SE, Chaudhuri RR, Harris D, Angen O, Aragon V, 
430

431

432

433

434

435

436

437

438

439

440

441

442

443

444

445

446

447

448

449

450

451

452

453

454

455

456

457

458

459

460

461

462

463

464

465

Parkhill J, Langford PR, Rycroft AN, Wren BW, Tucker AW, Maskell DJ, and Consortium BRT. 2013. Gene content and diversity of the loci encoding biosynthesis of capsular polysaccharides of the 15 serovar reference strains of Haemophilus parasuis. Journal of Bacteriology 195:4264-4273. 10.1128/JB.00471-13

Howell KJ, Weinert LA, Peters SE, Wang J, Hernandez-Garcia J, Chaudhuri RR, Luan SL, Angen O, Aragon V, Williamson SM, Langford PR, Rycroft AN, Wren BW, Maskell DJ, and Tucker AW. 2017. "Pathotyping" multiplex PCR assay for Haemophilus parasuis: a tool for prediction of virulence. Journal of Clinical Microbiology 55:2617-2628. 10.1128/JCM.02464-16

Kavanova L, Prodelalova J, Nedbalcova K, Matiasovic J, Volf J, Faldyna M, and Salat J. 2015. Immune response of porcine alveolar macrophages to a concurrent infection with porcine reproductive and respiratory syndrome virus and Haemophilus parasuis in vitro. Veterinary Microbiology 180:28-35. 10.1016/j.vetmic.2015.08.026

Kielstein P, and Rapp-Gabrielson VJ. 1992. Designation of 15 serovars of Haemophilus parasuis on the basis of immunodiffusion using heat-stable antigen extracts. Journal of Clinical Microbiology 30:862-865.

Kielstein P, Rosner H, and Mueller W. 1991. Typing of heat-stable soluble Haemophilus parasuis antigen by means of agar gel precipitation and the dot-blot procedure. Journal of Veterinary Medicine B: Infectious Diseases and Veterinary Public Health 38.

Li J, Wang S, Li C, Wang C, Liu Y, Wang G, He X, Hu L, Liu Y, Cui M, Bi C, Shao Z, Wang X, Xiong T, Cai X, Huang L, and Weng C. 2017. Secondary Haemophilus parasuis infection enhances highly pathogenic porcine reproductive and respiratory syndrome virus (HP-PRRSV) infection-mediated inflammatory responses. Veterinary Microbiology 204:35-42. 10.1016/j.vetmic.2017.03.035

Li JX, Jiang P, Wang Y, Li YF, Chen W, Wang XW, and Li P. 2009. Genotyping of Haemophilus parasuis from diseased pigs in China and prevalence of two coexisting virus pathogens. Preventive Veterinary Medicine 91:274-279. 10.1016/j.prevetmed.2009.06.004

Lin CN, Lin WH, Hung LN, Wang SY, and Chiou MT. 2013. Comparison of viremia of type II porcine reproductive and respiratory syndrome virus in naturally infected pigs by zip nucleic acid probe-based real-time PCR. BMC Veterinary Research 9:181. 10.1186/17466148-9-181

Luppi A, Bonilauri P, Dottori M, Iodice G, Gherpelli Y, Merialdi G, Maioli G, and Martelli P. 2013. Haemophilus parasuis serovars isolated from pathological samples in Northern Italy. Transboundary and Emerging Diseases 60:140-142. 10.1111/j.18651682.2012.01326.x 
466

467

468

469

470

471

472

473

474

475

476

477

478

479

480

481

482

483

484

485

486

487

488

489

490

491

492

493

494

495

496

497

498

499

500

501

Ma L, Wang L, Chu Y, Li X, Cui Y, Chen S, Zhou J, Li C, Lu Z, Liu J, and Liu Y. 2016. Characterization of Chinese Haemophilus parasuis isolates by traditional serotyping and molecular serotyping methods. PloS One 11:e0168903. 10.1371/journal.pone.0168903

Miniats OP, Smart NL, and Ewert E. 1991a. Vaccination of gnotobiotic primary specific pathogen-free pigs against Haemophilus parasuis. Canadian Journal of Veterinary Research 55:33-36.

Miniats OP, Smart NL, and Rosendal S. 1991b. Cross protection among Haemophilus parasuis strains in immunized gnotobiotic pigs. Canadian Journal of Veterinary Research 55:3741.

Moller K, and Kilian M. 1990. V factor-dependent members of the family Pasteurellaceae in the porcine upper respiratory tract. Journal of Clinical Microbiology 28:2711-2716.

Morozumi T, and Nicolet J. 1986. Some antigenic properties of Haemophilus parasuis and a proposal for serological classification. Journal of Clinical Microbiology 23:1022-1025.

Nielsen R. 1993. Pathogenicity and immunity studies of Haemophilus parasuis serotypes. Acta Veterinaria Scandinavica 34:193-198.

Oliveira S, Blackall PJ, and Pijoan C. 2003. Characterization of the diversity of Haemophilus parasuis field isolates by use of serotyping and genotyping. American Journal of Veterinary Research 64:435-442.

Olvera A, Ballester M, Nofrarias M, Sibila M, and Aragon V. 2009. Differences in phagocytosis susceptibility in Haemophilus parasuis strains. Veterinary Research 40:24. $10.1051 /$ vetres/2009007

Olvera A, Calsamiglia M, and Aragon V. 2006a. Genotypic diversity of Haemophilus parasuis field strains. Applied and Environmental Microbiology 72:3984-3992. 10.1128/AEM.02834-05

Olvera A, Cerda-Cuellar M, and Aragon V. 2006b. Study of the population structure of Haemophilus parasuis by multilocus sequence typing. Microbiology 152:3683-3690. 10.1099/mic.0.29254-0

Olvera A, Segales J, and Aragon V. 2007. Update on the diagnosis of Haemophilus parasuis infection in pigs and novel genotyping methods. Veterinary Journal 174:522-529. 10.1016/j.tvj1.2006.10.017

Palzer A, Haedke K, Heinritzi K, Zoels S, Ladinig A, and Ritzmann M. 2015. Associations among Haemophilus parasuis, Mycoplasma hyorhinis, and porcine reproductive and respiratory syndrome virus infections in pigs with polyserositis. Canadian Veterinary Journal 56:285-287.

Pina S, Olvera A, Barcelo A, and Bensaid A. 2009. Trimeric autotransporters of Haemophilus parasuis: generation of an extensive passenger domain repertoire specific for pathogenic 
502

503

504

505

506

507

508

509

510

511

512

513

514

515

516

517

518

519

520

521

522

523

524

525

526

527

528

529

530

531

532

533

534

535

536 537

strains. Journal of Bacteriology 191:576-587. 10.1128/JB.00703-08

Rafiee M, Bara M, Stephens CP, and Blackall PJ. 2000. Application of ERIC-PCR for the comparison of isolates of Haemophilus parasuis. Australian Veterinary Journal 78:846849.

Rapp-Gabrielson VJ, and Gabrielson DA. 1992. Prevalence of Haemophilus parasuis serovars among isolates from swine. American Journal of Veterinary Research 53:659-664.

Rubies X, Kielstein P, Costa L, Riera P, Artigas C, and Espuna E. 1999. Prevalence of Haemophilus parasuis serovars isolated in Spain from 1993 to 1997. Veterinary Microbiology 66:245-248. 10.1016/S0378-1135(99)00007-3

Segales J, Domingo M, Solano GI, and Pijoan C. 1999. Porcine reproductive and respiratory syndrome virus and Haemophilus parasuis antigen distribution in dually infected pigs. Veterinary Microbiology 64:287-297.

Smart NL, and Miniats OP. 1989. Preliminary assessment of a Haemophilus parasuis bacterin for use in specific pathogen free swine. Canadian Journal of Veterinary Research 53:390-393.

Solano GI, Segales J, Collins JE, Molitor TW, and Pijoan C. 1997. Porcine reproductive and respiratory syndrome virus (PRRSv) interaction with Haemophilus parasuis. Veterinary Microbiology 55:247-257.

Suradhat S, and Thanawongnuwech R. 2003. Upregulation of interleukin-10 gene expression in the leukocytes of pigs infected with porcine reproductive and respiratory syndrome virus. Journal of General Virology 84:2755-2760. 10.1099/vir.0.19230-0

Tadjine M, Mittal KR, Bourdon S, and Gottschalk M. 2004. Development of a new serological test for serotyping Haemophilus parasuis isolates and determination of their prevalence in North America. Journal of Clinical Microbiology 42:839-840.

Takahashi K, Naga S, Yagihashi T, Ikehata T, Nakano Y, Senna K, Maruyama T, and Murofushi J. 2001. A cross-protection experiment in pigs vaccinated with Haemophilus parasuis serovars 2 and 5 bacterins, and evaluation of a bivalent vaccine under laboratory and field conditions. Journal of Veterinary Medical Science 63:487-491. 10.1292/jvms.63.487

Turni C, and Blackall P. 2007. Comparison of sampling sites and detection methods for Haemophilus parasuis. Australian Veterinary Journal 85:177-184. 10.1111/j.17510813.2007.00136.x

Turni C, and Blackall PJ. 2005. Comparison of the indirect haemagglutination and gel diffusion test for serotyping Haemophilus parasuis. Veterinary Microbiology 106:145-151. 10.1016/j.vetmic.2004.12.019

Turni C, and Blackall PJ. 2010. Serovar profiling of Haemophilus parasuis on Australian farms by sampling live pigs. Australian Veterinary Journal 88:255-259. 10.1111/j.1751- 
538

539

540

541

542

543

544

545

546

547

548

549

550

551

552

553

554

555

556

557

558

559

560

561

562

563

564

565

566

567

568

569

570 571

0813.2010.00592.x

Vahle JL, Haynes JS, and Andrews JJ. 1995. Experimental reproduction of Haemophilus parasuis infection in swine: clinical, bacteriological, and morphologic findings. Journal of Veterinary Diagnostic Investigation 7:476-480. 10.1177/104063879500700409

Vahle JL, Haynes JS, and Andrews JJ. 1997. Interaction of Haemophilus parasuis with nasal and tracheal mucosa following intranasal inoculation of cesarean derived colostrum deprived (CDCD) swine. Canadian Journal of Veterinary Research 61:200-206.

Zhang B, Tang C, Liao M, and Yue H. 2014. Update on the pathogenesis of Haemophilus parasuis infection and virulence factors. Veterinary Microbiology 168:1-7. 10.1016/j.vetmic.2013.07.027

Figure legends

Figure 1 Distribution of Haemophilus parasuis serovars according to lesion pattern. Serositis only: animals were diagnosed with $H$. parasuis positive serosal lesions. Pulmonary lesion only: animals were diagnosed with $H$. parasuis positive pulmonary lesions. Data were analyzed by Fisher's exact test and variables were considered significant at a 0.05 level (twosided).

Figure 2 Haemophilus parasuis isolation proportion of 204 lesions of 108 pathological diagnosed cases.

Fisher's exact test was used to compare the frequency of $H$. parasuis isolation lesions. $P$ value $<$ 0.05 was considered a significant difference.

Figure 3 Molecular serotyping results with or without sequence results for 133 Haemophilus parasuis isolates.

\section{Supplementary file}

Supplementary Figure 1 Gross meningeal lesion in H. parasuis infected pigs.

Supplementary Figure 2 Histopathological suppurative meningitis lesion in H. parasuis infected pigs.

Supplementary Figure 3 Gross pleural and peritoneal lesions in H. parasuis infected pigs. 
572 Supplementary Figure 4 Gross epicardial lesion in H. parasuis infected pigs.

573

574 Supplementary Figure 5 Gross joint synovial cavity lesion in H. parasuis infected pigs.

575

576 Supplementary Figure 6 Histopathological fibrinous serositis lesion in H. parasuis infected 577 pigs.

578

579

Supplementary Figure 7 Gross lung lesion in H. parasuis infected pigs.

580

581

Supplementary Figure 8 Histopathological pulmonary lesion in $H$. parasuis infected pigs.

582

583

Supplementary Figure 9 Histopathological fibrous serositis lesion in H. parasuis infected pigs.

584

585

Supplementary Figure 10 Histopathological meningitis lesion in H. parasuis infected pigs.

586

587

Supplementary Figure 11 Band patterns of molecular serotyping mPCR for Haemophilus

588 parasuis.

589

Lane M: 50 bp DNA Ladder, lane S5: serovar 5 or 12, lane G2: molecular serotyping non-

590 typable group 2, lane S4: serovar 4, lane S9: serovar 9, lane G1: molecular serotyping non-

591 typable group 1, lane NC: negative control.

592

593

Supplementary Figure 12 Band patterns of molecular serotyping mPCR for Haemophilus 594 parasuis.

595 Lane M: 50 bp DNA Ladder, lane S4: serovar 4, lane S5: serovar 5 or 12, lane G2: molecular serotyping non-typable group 2, lane S14: serovar 14, lane G1: molecular serotyping non-typable group 1, lane NC: negative control.

Supplementary Figure 13 Band patterns of molecular serotyping mPCR for Haemophilus parasuis.

601 Lane M: 50 bp DNA Ladder, lane S5: serovar 5 or 12, lane S4: serovar 4, lane G1: molecular

602 serotyping non-typable group 1, lane G2: molecular serotyping non-typable group 2, lane NC:

603 negative control.Histopathological bronchopneumonia lesion in H. parasuis infected pigs.

604

605 Supplementary Figure 14 Band patterns of molecular serotyping mPCR for Haemophilus 606 parasuis. 
607 Lane M: 50 bp DNA Ladder, lane G1: molecular serotyping non-typable group 1, lane S4:

608 serovar 4, lane S5: serovar 5 or 12, lane S14: serovar 14, lane G4: molecular serotyping non-

609 typable group 4, lane G3: molecular serotyping non-typable group 3, lane NC: negative control.

610

611 Supplementary Figure 15 Band patterns of molecular serotyping mPCR for Haemophilus

612 parasuis.

613 Lane M: 50 bp DNA Ladder RTU (GeneDireX), lane G2: molecular serotyping non-typable

614 group 2, lane S4: serovar 4, lane S5: serovar 5 or 12, lane G1: molecular serotyping non-typable

615 group 1, lane G3: molecular serotyping the non-typable group 3, lane NC: negative control.

\section{Ethics and consent to participate}

617 The study did not involve any animal experiment. The Institutional Animal Care and Use

618 Committee (IACUC) of National Pingtung University of Science and Technology did not deem

619 it necessary for this research group to obtain formal approval to conduct this study.

620

621 Consent to publish

622 Not applicable.

623

624 Competing interest

625 The authors declare that they have no competing interests.

626

627 Funding

628 No funding was obtained for this study. 


\section{Authors' contributions}

631 Wei-Hao Lin, Chao-Nan Lin and Ming-Tang Chiou designed this study. Wei-Hao Lin performed

632 the laboratory experiments, analyzed data and wrote the manuscript. Hsing-Chun Shih assisted

633 the laboratory experiments. Chuen-Fu Lin, Cheng-Yao Yang, Yung-Fu Chang, Chao-Nan Lin

634 and Ming-Tang Chiou proofread and edited the manuscript.

635

\section{Availability of data and materials}

637 All the data supporting our findings is contained within the manuscript.

638

\section{Acknowledgements}

640 This study was supported by the Animal Disease Diagnostic Center, College of Veterinary

641 Medicine, National Pingtung University of Science and Technology, Taiwan. We appreciate the

642 following people for their help with this study: swine veterinarians at the Animal Disease

643 Diagnostic Center for assistance in necropsy, Ying-Xiu Lian for assistance in viral nucleic acid

644 detection and Qiong-Yi Huang to demonstrate bacterial preservation. Swine veterinarians who

645 assisted in this study: Guan-Shiuan Su, Chih-Chung Chang, Shu-Wei Chang, Kuan-Lin Li, Ling-

646 Fang Wang, Guang-Ting Tsai, Ni-Jyun Ke, Ting-Han Lin, Sheng-Yuan Wang, Hong Liu, Jia-

647 Liang Hong, Joan Wang and Yu-Hsuan Chen. 


\section{Figure 1}

Distribution of Haemophilus parasuis serovars according to lesion pattern.

Distribution of Haemophilus parasuis serovars according to lesion pattern. Serositis only: animals were diagnosed with $H$. parasuis positive serosal lesions. Pulmonary lesion only: animals were diagnosed with $H$. parasuis positive pulmonary lesions. Data were analyzed by Fisher's exact test and variables were considered significant at a 0.05 level (two-sided).

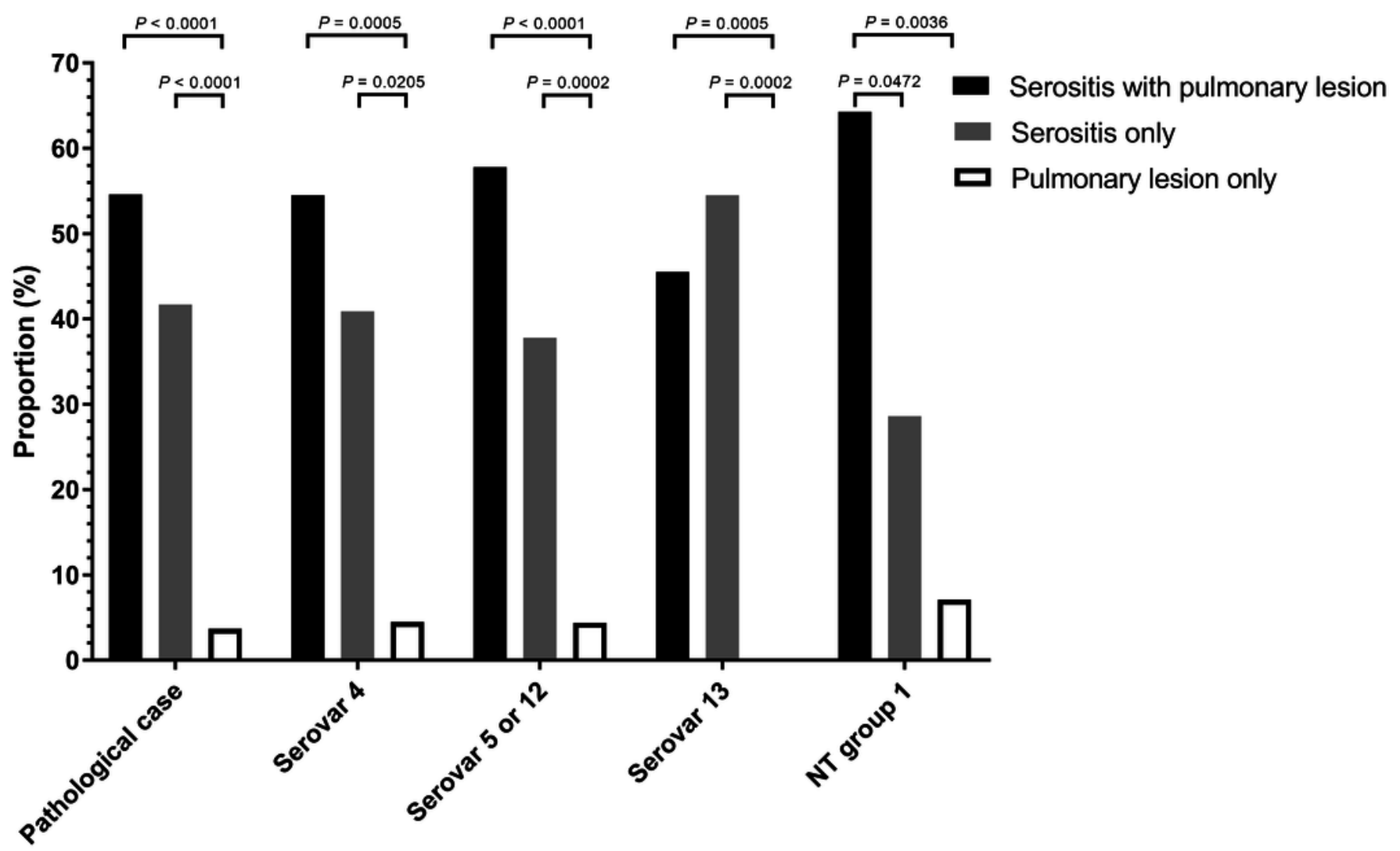




\section{Figure 2}

Haemophilus parasuis isolation proportion of 204 lesions of 108 pathological diagnosed cases.

Haemophilus parasuis isolation proportion of 204 lesions of 108 pathological diagnosed cases. Fisher's exact test was used to compare the frequency of $H$. parasuis isolation lesions. $P$ value $<0.05$ was considered a significant difference.
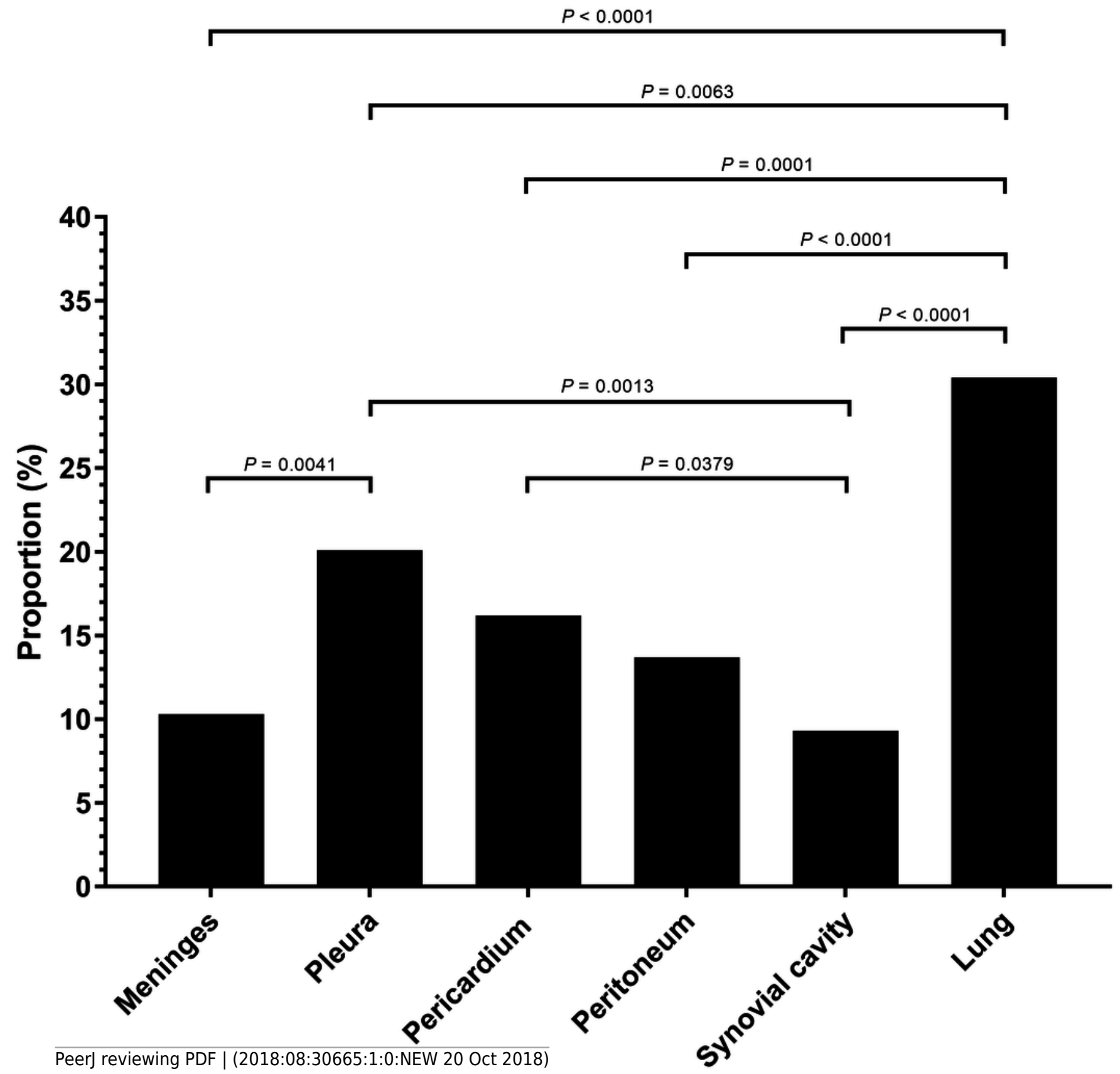
Figure 3

Molecular serotyping results with or without sequence results for 133 Haemophilus parasuis isolates.

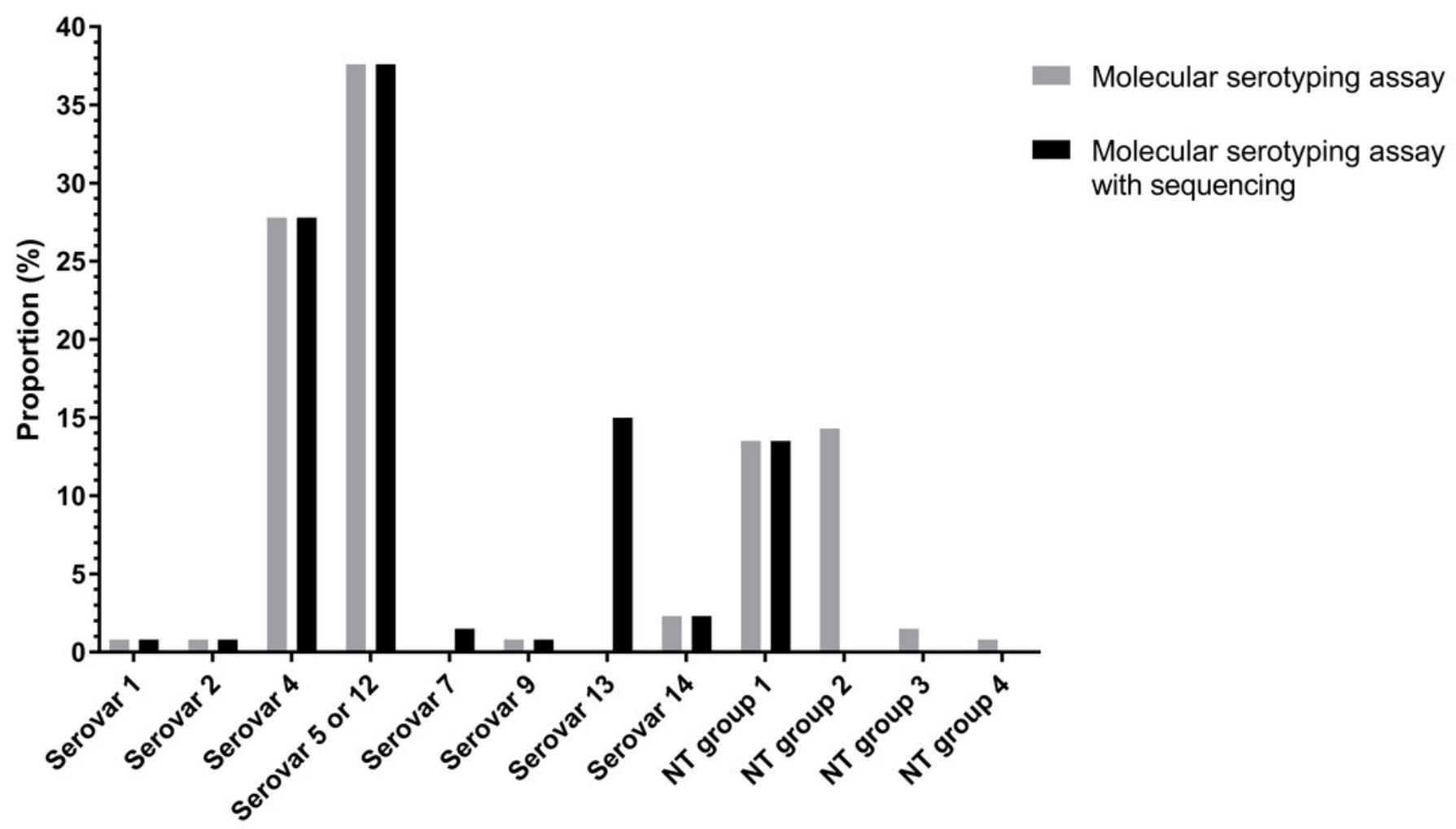




\section{Table $\mathbf{1}$ (on next page)}

Product size by molecular serotyping assay 
1 Table 1 Product size by molecular serotyping assay (Howell et al. 2015)

\begin{tabular}{|c|c|c|c|c|c|c|}
\hline Gene & Serovar & $\begin{array}{l}\text { Product size in } \\
\text { the original } \\
\text { publication (bp) }\end{array}$ & $\begin{array}{c}\text { Product size (bp) } \\
\text { predicted by BLAST }\end{array}$ & $\begin{array}{l}\text { Aligned sequence } \\
\text { accession number }\end{array}$ & $\begin{array}{c}\text { Product size (bp) measured by } \\
\text { Bio-1D software }\end{array}$ & $\begin{array}{l}\text { Product size }(b p) \\
\text { according to sequenc }\end{array}$ \\
\hline$f u n B$ & 1 & 180 & 183 & CL120103 & 184 & 183 \\
\hline$w z x$ & $2 \dagger$ & 295 & 294 & CL120103 & N/A & N/A \\
\hline$g l y C$ & $3 \dagger$ & 610 & 618 & KC795327.1 & $\mathrm{N} / \mathrm{A}$ & N/A \\
\hline$w c i P$ & 4 & 320 & 349 & КC795356.1 & 350 & 349 \\
\hline$w c w K$ & 5 or 12 & 450 & 468 & KC795341.1 & 469 & 468 \\
\hline gltI & $6 \dagger$ & 360 & 378 & KC795372.1 & $\mathrm{N} / \mathrm{A}$ & $\mathrm{N} / \mathrm{A}$ \\
\hline funQ & $7 \dagger$ & 490 & 499 & СР009158.1 & $\mathrm{N} / \mathrm{A}$ & $\mathrm{N} / \mathrm{A}$ \\
\hline$s c d A$ & $8 †$ & 650 & 634 & KC795411.1 & N/A & N/A \\
\hline funV & 9 & 710 & 676 & КC795429.1 & 675 & 676 \\
\hline funX & $10 \dagger$ & 790 & 784 & KC795448.1 & N/A & N/A \\
\hline$a m t A$ & $11 \dagger$ & 890 & 883 & КC795474.1 & N/A & N/A \\
\hline gltP & $13 \dagger$ & 840 & 836 & KF841370.1 & N/A & N/A \\
\hline fun $A B$ & 14 & 730 & 710 & KC795520.1 & 708 & 710 \\
\hline funI & $15 \dagger$ & 550 & 550 & KC795537.1 & N/A & N/A \\
\hline HPS_219690793 & All & 275 & 276 & СР020085.1 & 276 & 276 \\
\hline
\end{tabular}

2 †This serotype was not detected in this study. 
Table 2 (on next page)

Unexpected products of serotyping multiplex PCR 
1 Table 2 Unexpected products of serotyping multiplex PCR

\begin{tabular}{|c|c|c|c|c|}
\hline $\begin{array}{l}\text { Molecular } \\
\text { serotyping } \\
\text { non-typable } \\
\text { isolate }\end{array}$ & $\begin{array}{l}\text { Serovar according to } \\
\text { sequence }\end{array}$ & $\begin{array}{l}\text { Product size (bp) measured by Bio- } \\
\text { 1D software }\end{array}$ & $\begin{array}{c}\text { Product size (bp) according } \\
\text { to sequence }\end{array}$ & $\begin{array}{c}\text { Amplified } \\
\text { primer }\end{array}$ \\
\hline \multirow[t]{2}{*}{ Group 1} & \multirow[t]{2}{*}{ Unknown† } & Noneł & None & None \\
\hline & & 300 & 297 & S13F, S14R \\
\hline \multirow[t]{2}{*}{ Group 2} & \multirow[t]{2}{*}{ Serovar 13} & 830 & 836 & $\mathrm{~S} 13$ \\
\hline & & 1000 & N/A§ & N/A \\
\hline \multirow{2}{*}{ Group 3} & \multirow{2}{*}{ Serovar 7} & 500 & 499 & $\mathrm{~S} 7$ \\
\hline & & 660 & N/A & N/A \\
\hline Group 4 & Serovar 13 & 300 & 297 & S13F, S14R \\
\hline
\end{tabular}

2 †Serovar could not be defined without any serovar-specific product sequence result.

$3 \mp$ There was no serovar-specific product.

4 §Cloning of serovar-specific product was failed. 